\title{
Hugo von Hofmannsthal et l'éloge du canon classique français. De l'exemplarité racinienne
}

Hugo von Hofmannsthal und das Lob des französischen klassischen Kanons

Hugo von Hofmannsthal and the praise of the French classical canon

\section{Audrey Giboux}

\section{(2) OpenEdition}

\section{Journals}

Édition électronique

URL : http://journals.openedition.org/ceg/5729

DOI : $10.4000 /$ ceg. 5729

ISSN : 2605-8359

Éditeur

Presses Universitaires de Provence

Édition imprimée

Date de publication : 1 octobre 2013

Pagination : 75-88

ISSN : 0751-4239

Référence électronique

Audrey Giboux, " Hugo von Hofmannsthal et l'éloge du canon classique français. De l'exemplarité racinienne », Cahiers d'Études Germaniques [En ligne], 65 | 2013, mis en ligne le 01 octobre 2019,

consulté le 02 décembre 2020. URL : http://journals.openedition.org/ceg/5729 ; DOI : https://doi.org/ $10.4000 /$ ceg. 5729 


\title{
Hugo von Hofmannsthal et l'éloge du canon classique français. De l'exemplarité racinienne
}

Audrey GIBOUX

\author{
Université Rennes 2
}

\section{Les aléas de l'exemplarité française}

$\mathrm{Si}$, en dépit de ses propres doutes face à l'idée de moderne ${ }^{1}$, Hofmannsthal est considéré comme une figure emblématique de la modernité viennoise, son rapport à cette valeur esthétique conquérante qu'est la modernité s'est souvent éprouvé de manière ambiguë, voire douloureuse : en témoigne notamment la célèbre Lettre de 1902, dite « de Lord Chandos », dans laquelle le personnage fictif, incarnation de la culture humaniste de la Renaissance, établit un diagnostic désenchanté, volontairement anachronique, sur les possibilités mêmes du langage et de l'expression littéraire, voués à la dispersion ; en témoigne également la difficulté du poète et dramaturge qu'est Hofmannsthal à apprivoiser certaines des formes génériques canoniques, le conduisant à laisser inachevé Andreas, le roman de formation auquel il travaille de 1907 à 1927, faute de parvenir à circonscrire ses proliférations ${ }^{2}$.

Face au risque de désagrégation que suppose à ses yeux la tentation moderne, le recours au classicisme apparaît comme un rempart, ce qu'attestent dès le tournant des $\mathrm{XIX}^{\mathrm{e}}$ et $\mathrm{XX}^{\mathrm{e}}$ siècles ses réécritures dramatiques puisées, à travers le filtre d'œuvres plus récentes, à la source de

${ }^{1}$ Voir notamment « Gabriele d'Annunzio » [1893], in Hugo von HofmannSTHAL, Reden und Aufsätze, Band I, hrsg. von Bernd Schoeller und Ingeborg Beyer-Ahlert in Beratung mit Rudolf Hirsch, Frankfurt am Main, Fischer Taschenbuch, 1979-1980, p. 175-176. Dans la suite de cet article, les trois volumes de Reden und Aufsätze seront désignés par les abréviations RA I, RA II et RA III.

2 « Ein Brief», in Hugo von Hofmannsthal, Sämtliche Werke. Kritische Ausgabe, Band XXXI, Erfundene Gespräche und Briefe, hrsg. von Ellen Ritter, Frankfurt am Main, Fischer, 1991, p. 45-55; Andreas, in Sämtliche Werke, Band XXX, Roman. Biographie, aus dem Nachlass hrsg. von Manfred Pape, Frankfurt am Main, Fischer, 1982, p. 5-218. 
la tradition antique ${ }^{3}$. Son œuvre critique révèle aussi son attachement aux traditions littéraires dans leur diversité et rend de fréquents hommages aux chefs-d'œuvre européens ou à cette littérature universelle dont Goethe avait espéré et prophétisé l'avènement ${ }^{4}$. La modernité problématique de l'œuvre de Hofmannsthal revêt un fondement paradoxal, puisqu'il revendique une forme de classicisme, essentiellement par la référence à la littérature française.

Cette posture, dans un contexte diplomatique profondément dégradé entre la France et les pays germaniques, paraît originale et même provocatrice dans la mesure où, depuis la fin du XVIII ${ }^{\mathrm{e}}$ siècle, les plus grands auteurs allemands se sont souvent montrés réticents envers l'imitation du modèle français ${ }^{5}$. La position francophile de Hofmannsthal trouve à vrai dire un précédent dans la nouvelle impulsion donnée par Gottsched au théâtre germanique, en amont de ces critiques du paradigme français ${ }^{6}$; dans le sillage gottschédien, avant que n'émerge la volonté patriotique, voire gallophobe, de se démarquer d'un modèle rapidement considéré comme un repoussoir social et éthique, les dramaturges des Lumières se sont efforcés de réformer, de moraliser et de rendre le théâtre allemand capable de rivaliser avec les autres scènes européennes, grâce à une pratique militante de l'adaptation de pièces françaises. La classicisation de la comédie allemande des Lumières, par la référence au canon français, repose sur un processus de sélection, d'assimilation, d'actualisation et de germanisation de topiques et techniques dramaturgiques qui, poursuivant la quête d'une identité nationale problématique et d'un modèle de civilité bourgeoise typiquement allemand, contribue à cristalliser de durables stéréotypes imagologiques associés aux peuples français et allemand? ${ }^{7}$.

${ }^{3}$ Alkestis [1894], Elektra [1903], in Sämtliche Werke, Band VII, Dramen 5, hrsg. von Klaus E. Bohnenkamp und Mathias Mayer, Frankfurt am Main, Fischer, 1997 ; Ödipus und die Sphinx [1906], König Ödipus [1910], in Sämtliche Werke, Band VIII, Dramen 6, hrsg. von Wolfgang Nehring und Klaus E. Bohnenkamp, Frankfurt am Main, Fischer, 1983.

${ }^{4}$ Johann Peter ECKermann, Gespräche mit Goethe in den letzten Jahren seines Lebens, in Johann Wolfgang von GoETHE, Sämtliche Werke nach Epochen seines Schaffens, Band XIX, hrsg. von Heinz Schlaffer, München, Hanser, 1986, p. 207, 237 et 254.

Voir notamment Gotthold Ephraim LeSsiNG, Hamburgische Dramaturgie [1767-1769], in Werke, Band IV, Dramaturgische Schriften, hrsg. von Herbert G. Göpfert, München, Hanser, 1973, p. 601-619; Johann Wolfgang von GoETHE, «Zum Schäkespears [sic] Tag » [1771], in Sämtliche Werke, Band I.2, Der junge Goethe 1757-1775, hrsg. von Gerhard Sauder, München, Hanser, 1987 ; Friedrich SCHILLER, "Über die tragische Kunst » [1792], " Über das Pathetische » [1793], in Werke und Briefe, Band VIII, Theoretische Schriften, hrsg. von RolfPeter Janz, Frankfurt am Main, Deutscher Klassiker Verlag, 1992, respectivement p. 251-275 et 423-451 ; August Wilhelm Schlegel, Comparaison entre la Phèdre de Racine et celle d'Euripide (et autres textes), édition présentée par Jean-Marie Valentin, Arras, Artois Presses Université, 2013, p. 99-183.

6 Johann Christoph GOTTSCHED, Versuch einer critischen Dichtkunst [1730], in Ausgewählte Werke, Band VI.1, Erster allgemeiner Theil und Band VI.2, Anderer besonderer Theil, hrsg. von Joachim Birke und Brigitte Birke, Berlin / New York, De Gruyter, 1973.

7 Voir Elsa JAUBERT, De la scène au salon. Le Modèle français dans la comédie allemande des Lumières, Paris, Presses de l’Université Paris-Sorbonne, 2012. 
C'est cette même ambivalence, entre admiration déclarée pour la littérature française et désir de fonder une nouvelle scène germanophone, qui préside aux ambitions théâtrales de Hofmannsthal. À une époque de troubles exacerbés par la Première Guerre mondiale, le dramaturge autrichien éprouve plus que jamais le besoin de réhabiliter la littérature française aux yeux de ses contemporains : la comparaison des littératures française et autrichienne participe sous sa plume d'un renoncement à l'absolutisation des génies nationaux.

\section{Une lecture politique et cosmopolite des enjeux littéraires}

Lorsque la guerre éclate, Hofmannsthal cherche à restaurer une vision idéaliste et humaniste de l'Europe : de même que la Renaissance a réinvesti l'héritage antique dans la littérature moderne, c'est à une nouvelle Europe des lettres qu'est confiée la mission de préserver un patrimoine culturel transnational ${ }^{8}$. Le conflit rend en effet cruciale la nécessité de distinguer les aspirations autrichiennes de celles des Allemands ${ }^{9}$; Hofmannsthal poursuit sa réflexion dialogique sur la spécificité autrichienne dans l'espace germanique en soulignant la parenté des Autrichiens et des Français dans la primauté accordée au fait social ${ }^{10}$. Sa position oscille ainsi constamment entre une assimilation de l'Autriche à l'identité culturelle allemande ${ }^{11}$ et la revendication de la singularité de son pays ${ }^{12}$.

Sa profession de foi xénophile suppose la capacité non démentie de la France à jouer un rôle de référence civilisatrice universelle et de modèle alternatif pour l'austriacité ; son éloge de la culture classique française, déjà souvent présent sous sa plume, s'intensifie au tournant de l'année 1916, lorsque ses ambitions pacificatrices prennent définitivement le pas sur ses éphémères velléités patriotiques. Dans cette logique où la constitution identitaire présuppose un discours sur l'altérité, la littérature française se voit idéalisée en raison de la communion qu'elle établit entre la parole des auteurs, pleinement intégrés à la société, et celle du peuple, garantissant la transmission de la culture de siècle en siècle; à l'inverse, les pays germanophones ne possèdent pas, aux yeux de Hofmannsthal, de véritable tradition littéraire, capable d'exprimer le génie de la nation et de révéler les liens qui unissent la communauté des locuteurs à travers l'histoire ${ }^{13}$. La

8 « Bücher für diese Zeit » [1914], in RA II, p. 367-374 ; « Die Idee Europa » [1917], ibid., p. $43-54$.

9 «Wir Österreicher und Deutschland» [1915], in RA II, p. 390.

10 «Preuße und Österreicher. Ein Schema » [1917], in RA II, p. 459-461.

" Ein deutscher Homer von heute. Die Odyssee. Deutsch von Rudolf Alexander Schröder» [1912], in RA I, p. 412.

${ }^{12}$ «Ferdinand von Saar, Schloss Kostenitz » [1892], in RA I, p. 139 ; « Rede auf Grillparzer für die deutsche Grillparzer-Gedenkfeier zu Hannover, den 7. Mai $1922 »$, in RA II, p. 87.

${ }_{13}$ " Gemüt. Der Bedeutungswandel eines deutschen Wortes» [1925], in RA III, p. 56-57. 
langue littéraire qu'il appelle de ses vœux est alors décrite comme le véritable corps spirituel de la nation :

\begin{abstract}
[La nation française], de fait, possède une littérature au vrai sens du terme. Les grands textes parus depuis le début de l'ère moderne, c'est-à-dire depuis environ trois cent cinquante ans, semblent toujours vivants. Les médiocres, qui entretiennent avec ceux-là un rapport de claire infériorité, replongent dans l'obscurité au bout de quelque temps et rejaillissent dans de nouvelles formes pleines d'esprit. Même les petits textes, destinés aux contemporains, ne manquent pas, tout le temps qu'ils durent, d'une certaine dignité grâce au soin mis à rechercher une langue pure et à rendre les pensées de façon claire, ordonnée et compréhensible. ${ }^{14}$
\end{abstract}

C'est le principe même de la norme linguistique fixée par l'Académie française qui, d'après Hofmannsthal, assure la cohésion de la nation autour d'un idiome commun, tout en laissant s'exprimer les tendances les plus antagonistes des locuteurs, aristocratiques ou égalitaristes, révolutionnaires ou conservatrices. Cet idiome est ainsi le reflet direct de la sociabilité à la française. Hofmannsthal incrimine par là les carences de la littérature allemande, qui la privent de toute efficacité sociale et la conduisent à une prostration qui n'est que le corollaire de sa quête de transcendance : la norme d'une langue classique et la contrainte créatrice, capables de conjurer les dérives et d'exhausser les potentialités de la posture plus romantique de l'inspiration. Si la langue française est une langue sociale, c'est parce qu'elle porte une fonction civilisatrice, une charge critique et politique, une conscience historique qui lui donnent une emprise directe sur la réalité :

La notion d'espace qui émane de cet ensemble spirituel est identique à l'espace spirituel que la nation occupe à ses propres yeux et aux yeux du monde entier. Rien n'a de réalité dans la vie politique de la nation qui ne serait présent dans sa littérature sous forme d'esprit ; cette littérature débordante de vie et sans rêves ne contient rien qui ne se réalise dans la vie de la nation. Une dignité sans pareille se répand sur les hommes de lettres dans ce « paradis des paroles ». ${ }^{15}$

L'espace défini par la langue ne se présente donc nullement comme un territoire national, mais comme un ensemble symbolique animé par une

\footnotetext{
14 «Das Schrifttum als geistiger Raum der Nation» [1926], in RA III, p. 24-26: « [Die französische Nation] nun besitzt eine Literatur im wahren Sinne des Wortes. Das Große, seit Beginn der neueren Ära, das ist seit etwa dreihundertfünfzig Jahren, Hervorgetretene erscheint fortwirkend. Das Mittlere, zu jenem Großen in klar abgestuftem Verhältnis, tritt nach gemessener Zeit ins Dunkel zurück und steigt in neuen geistreichen Formen wieder hervor Selbst das Geringe, für den Tag Bestimmte, nimmt für die Spanne seiner Wirksamkeit teil an einer gewissen Würde durch die Sorgfalt, mit welcher es eine reine Sprache anstrebt und die Gedanken klar und wohlgeordnet und fasslich wiedergeben will. »

15 Ibid., p. 27-28 : «Der Raumbegriff, der aus diesem geistigen Ganzen emaniert, ist identisch mit dem Geistesraum, den die Nation in ihrem eigenen Bewusstsein und in dem der Welt einnimmt. Nichts ist im politischen Leben der Nation Wirklichkeit, das nicht in ihrer Literatur als Geist vorhanden wäre, nichts enthält diese lebensvolle, traumlose Literatur, das sich nicht im Leben der Nation verwirklichte. Auf den Literaten in diesem „Paradies der Worte“ strahlt eine Würde ohnegleichen. »
} 
mémoire et une littérature communes. Au sein de l'espace linguistique français, que Hofmannsthal représente comme une totalité close et harmonieuse, même le plus médiocre des journalistes prend place, selon lui, aux côtés de Bossuet et de La Bruyère ; tout instituteur est le compagnon de Montaigne, Molière et La Fontaine ; et Voltaire comme Montesquieu parlent encore pour tous les Français du XX $\mathrm{XX}^{\mathrm{e}}$ siècle, dans chacune de leurs prises de parole. L'attention de Hofmannsthal se porte alors vers le manque d'unanimité langagière dont est victime à ses yeux le peuple allemand.

\section{De la nécessité d'un répertoire germanique et européen}

Hofmannsthal affirme que la fidélisation du public autour d'un répertoire germanophone concourrait à développer chez lui un sentiment d'unité : « c'est dans la langue que souffre ou fleurit l'esprit du peuple ${ }^{16}$. " Le sentiment d'appartenance nationale, indépendamment de tout nationalisme, ne peut s'édifier que dans et par l'écriture, force de synthèse qui agit de génération en génération. Si le classicisme est clairement défini sous sa plume à la fois comme période et critère esthétiques ${ }^{17}$, paradigme de représentation du monde ${ }^{18}$ et valeur axiologique des textes ${ }^{19}$, la reconnaissance des classiques de l'Autriche est, selon lui, plus difficile qu'en France, parce que moins institutionnalisée ${ }^{20}$, ce qui rend d'autant plus nécessaires l'appropriation des classiques étrangers et la lutte en faveur de la traduction des œuvres et de l'enseignement des langues étrangères ${ }^{21}$. C'est en intégrant le classicisme européen que l'Autriche doit conquérir une voix littéraire singulière et affirmer une pensée de la modernité marquée par une hybridité, sur le modèle d'un art vivant, en perpétuelle renaissance ${ }^{22}$.

Dans cet esprit de conciliation des peuples autour d'un passé culturel commun, Hofmannsthal initie la publication, chez Insel, d'une série consacrée aux grandes figures de l'histoire et de la littérature autrichiennes: l'objectif de cette entreprise, finalement écourtée, est de favoriser l'unité du peuple autrichien autour de sa littérature et de faire entendre en Europe la voix de l'Autriche ${ }^{23}$. Il salue de même la publication, toujours chez Insel, d'une anthologie des chefs-d'œuvre de la littérature européenne en langue originale, qui se veut un hommage aux belles-lettres et une réaffirmation de la vocation universaliste de la culture allemande. La présentation de la collection Bibliotheca mundi est l'occasion d'évoquer la nécessité d'identifier

\footnotetext{
${ }^{16}$ « Ankündigung des Verlages der Bremer Presse » [1922], in RA II, p. 177 : « Denn in der Sprache leidet oder blüht der Geist des Volkes. »

17 « Über Walther Brecht » [1926], in RA III, p. 76.

18 Aufzeichnungen [1891], in RA III, p. 320 et p. 323.

19 " Ein deutscher Homer von heute », in RA I, p. 412.

20 «Deutsche Erzähler» [1912], in RA I, p. 430-431.

${ }^{21}$ « Boykott fremder Sprachen? » [1914], in RA II, p. 351-355.

${ }^{22}$ Aufzeichnungen [1891], in RA III, p. 323.

23 "Österreichische Bibliothek. Eine Ankündigung » [1915], in RA II, p. 432-439.
} 
les classiques de la littérature, par-delà les frontières politiques ; soulignant la lenteur avec laquelle les Français intègrent les grands auteurs à leur Panthéon et la constance avec laquelle, en revanche, ils maintiennent vivante leur gloire, Hofmannsthal indique combien un regard porté de l'étranger sur la littérature française peut jouer le rôle d'une postérité par anticipation, capable de filtrer plus rapidement que ne le font la critique et le public français les cuvres de valeur ${ }^{24}$.

Un répertoire théâtral nettement constitué est pour lui un élément de continuité spirituelle au fil des siècles, s'adressant, entre une exigence de classicisme et une vocation populaire, à un large public, comme c'est le cas en France. L'institution de la Comédie-Française lui semble en effet fonder sa légitimité incontestée sur son répertoire presque immuable de classiques, c'est-à-dire d'œuvres, d'esthétique classique ou plus moderne, identifiées par le public comme relevant d'un patrimoine théâtral national :

\begin{abstract}
Ils jouent leur Corneille, leur Racine et leur Molière depuis deux cents ou deux cent cinquante ans. C'est le fond immuable de leur répertoire. À cela sont venues s'ajouter maintes comédies des auteurs de moindre importance du XVIII ${ }^{\mathrm{e}}$ siècle, Regnard et Marivaux, puis tout au plus Beaumarchais et le peu qui reste de Voltaire au théâtre, ainsi qu'un petit nombre d'œuvres de la première moitié du $\mathrm{XIX}^{\mathrm{e}}$ siècle, Musset, Hernani et Ruy Blas de Victor Hugo ; et quelques pièces des analystes de la seconde moitié du siècle : Augier, Dumas, Sardou. Cet état de fait est infiniment stable, l'activité claire, évidente, il y a des chemins tout tracés. $^{25}$
\end{abstract}

Une semblable sélection de classiques dans le corpus du théâtre de langue allemande lui paraît incomparablement plus difficile. Si la définition du répertoire germanique semble plus délicate que celle du répertoire français, elle ne doit cependant pas se soumettre à la contingence de la mode :

Un répertoire, c'est le sens du mot, doit être une mine d'où l'on extrait jour après jour des valeurs sûres pour les porter à la lumière. Six générations consécutives, depuis Lessing jusqu'à nous, ont constitué un répertoire allemand qui peut présenter des couleurs diverses selon le lieu et le moment : mais enfin il ne doit être que teinté par l'esprit du lieu et du moment, non formé par lui. Il a reçu sa forme et son contenu du siècle de l'esprit allemand, de la grande époque qui s'étend de 1750 à 1850 ; celui qui conteste cela et prétend soumettre ce bien à

\footnotetext{
${ }^{24}$ «Bibliotheca mundi» [1921], in RA II, p. 136.

25 «Zur Krisis des Burgtheaters » [1918], in RA II, p. 243 : « Sie spielen ihren Corneille, ihren Racine und Molière seit zweihundert und zweihundertfünfzig Jahren. Das ist der unveränderliche Kern des Repertoires. Aus dem achtzehnten Jahrhundert ist etliches von den kleineren Komödiendichtern dazugetreten, Regnard und Marivaux, dann allenfalls Beaumarchais und das Wenige, das sich von Voltaire auf dem Theater erhalten hat; aus der ersten Hälfte des neunzehnten Jahrhunderts einiges Wenige von Musset, der Hernani und der Ruy Blas von Victor Hugo; aus der zweiten das eine oder andere Stück von den Analytikern: Augier, Dumas, Sardou. Wie unendlich beharrend ist diese Lage der Dinge, wie übersichtlich, wie selbstverständlich das Handeln, da sind geweiste Wege. »
} 
l'humeur du moment s'imagine manifestement qu'une grande nation vit au jour le jour, tel un mendiant, et il faut lui répliquer et lui résister en actes. ${ }^{26}$

C'est ainsi un nouveau classicisme qui pourrait présider à l'édification du répertoire germanique, et Hofmannsthal exprime l'espoir que la littérature autrichienne contemporaine soit capable de produire des œuvres à la fois neuves et suffisamment ancrées dans le classicisme des $\mathrm{XVII}^{\mathrm{e}}$ et $\mathrm{XVIII}^{\mathrm{e}}$ siècles pour se garantir une légitime reconnaissance de la part du public français ${ }^{27}$. Le répertoire des scènes allemandes ne saurait en tout état de cause se limiter à des œuvres germanophones : le mot d'ordre n'est pas la seule constitution d'un répertoire national, mais la préservation d'un répertoire transhistorique et transnational, qui puisse être joué sur toutes les scènes d'Europe $^{28}$.

\section{L'éloge du canon classique français : une prise de position cruciale en temps de guerre}

La France est, selon Hofmannsthal, la nation romane la plus proche de l'esthétique antique, comme si, parmi les peuples barbares, les Français étaient les seuls héritiers véritables des Grecs ; portant l'essence même de l'Antiquité «dans leur sang », ils semblent voir le monde à travers les mêmes yeux que les Grecs, vivre au même rythme qu'eux, être les seuls capables de saisir les contours de leur monde révolu et de reconnaître dans leur legs les esquisses majestueuses de Poussin, la finesse de Racine, l'acuité de Molière : le sens de la mesure, l'absence totale de flou et la clarté représentent ainsi pour Hofmannsthal les trois critères de l'esprit classique français ${ }^{29}$.

Ce constat s'accompagne d'une volonté pédagogique de faire redécouvrir les œuvres françaises au public germanophone : si l'éminence d'auteurs comme Molière, La Fontaine, La Bruyère, Boileau, Madame de Sévigné n'apparaît pas clairement au public allemand contemporain, il incombe aux écrivains allemands modernes, et à lui-même au premier chef, de l'aider à se

\footnotetext{
26 « Repertoire » [1925], in RA III, p. 173: « Repertorium, so sagt der Wortsinn, soll eine Fundgrube sein, daraus Tag um Tag das Altbewährte hervorgenommen und ans Licht gesteckt wird. Sechs Generationen nacheinander, von Lessing bis auf uns, haben ein deutsches Repertoire zusammengetragen, das je nach dem Ort und dem Zeitmoment verschieden gefärbt sein mag: aber es soll doch vom Orts- und vom Zeitgeist nur tingiert sein, nicht von ihm gestaltet. Seine Form und seinen Gehalt hat es von dem Jahrhundert deutschen Geistes empfangen, dem großen von 1750 bis 1850 währenden, und wer daran rührt und diesen Besitz dem launischen Moment unterworfen haben will, der ist offenbar des Glaubens, dass eine große Nation aus der Hand in den Mund lebe, wie ein Bettler, und ihm muss durch die Tat widersprochen und widerstanden werden. »

27 «Die Bedeutung unseres Kunstgewerbes für den Wiederaufbau. Ansprache an die Mitglieder des österreichischen Werkbundes » [1919], in RA II, p. 57-61.

28 «Reinhardt bei der Arbeit » [1923], in RA II, p. 295-296.

29 «Ein deutscher Homer von heute », in RA I, p. 413.
} 
déprendre de ses préjugés à l'égard de la littérature d'outre-Rhin ${ }^{30}$. Cette nécessité est justifiée par la parenté paradoxale que découvre Hofmannsthal entre le Grand Siècle français et l'Autriche contemporaine, comme en témoigne cette lettre datée du 24 août 1916, à l'heure où il éprouve douloureusement la crise des valeurs humanistes :

\begin{abstract}
Du reste, je lis presque chaque soir depuis des mois, avant de m'endormir, quelques pages de Madame de Sévigné, de La Fontaine, de La Bruyère, de La Rochefoucauld, de Molière, de Bossuet, et cette époque vraiment grande, extrêmement humaine aussi, me semble un vrai paradis. En outre, elle ne me paraît pas du tout si lointaine ou étrangère. En Autriche, on en est parfois étonnamment proche en ce qui concerne l'humeur générale des personnes justement les plus distinguées. Même le regard porté sur l'Antiquité me ravit parfois, comme si l'on voyait depuis une sombre vallée dans les Alpes des îles éternelles baignées de soleil. Je serais au désespoir si je ne pensais pas que, malgré tout, nous avançons à tâtons sur un chemin sombre, tortueux, sanglant, vers un nouvel humanisme. ${ }^{31}$
\end{abstract}

Le seul rempart contre une désagrégation des valeurs après la guerre, à contre-courant de la modernité régnante, semble être une réévaluation des classiques, propédeutique à la fondation d'un nouveau classicisme au $\mathrm{XX}^{\mathrm{e}}$ siècle. Hofmannsthal pointe l'absence d'une histoire commune, depuis le $\mathrm{XVI}^{\mathrm{e}}$ siècle, permettant d'unifier d'un point de vue spirituel les différentes composantes du peuple allemand. La littérature seule peut donc lui permettre de trouver sa physionomie propre et devenir le lieu où, " derrière chaque visage isolé qui nous regarde avec éminence et sincérité, à travers le fond obscur du miroir, perce encore le mystérieux visage national ${ }^{32} »$. C'est ce qu'a permis, dans l'histoire littéraire française, le processus de reconnaissance respectueuse des classiques du Grand Siècle ; le siècle de Voltaire, rappelle Hofmannsthal, a ainsi identifié a posteriori en Molière, La Fontaine, Racine et Pascal ses plus hautes figures tutélaires, tandis que la langue classique trouvait, dans une forme de réflexivité sur ses fondements littéraires, une pleine conscience de ses moyens, gage d'une souveraineté linguistique mondiale encore sensible au début $\mathrm{du} \mathrm{XX}^{\mathrm{e}}$ siècle. C'est ainsi que Hofmannsthal plaide pour une reconnaissance de l'équivalent germanique du

${ }^{30}$ Aufzeichnungen [1916], in RA III, p. 530.

31 Hugo von HOFMANNSTHAL-Helene von Nostiz, Briefwechsel, hrsg. von Oswalt von Nostiz, Frankfurt am Main, Fischer, 1965, p. 136-137 : «Im Übrigen lese ich seit Monaten fast jeden Abend vor dem Schlafen ein paar Seiten in der Sévigné, im La Fontaine, im La Bruyère, im La Rochefoucauld, im Molière, im Bossuet und finde diese wahrhaft große, auch höchst menschliche Zeit ein wahres Paradies. Auch scheint sie mir keineswegs gar so fern oder fremd. In Österreich ist man ihr, was die allgemeine Atmosphäre gerade der besten Leute betrifft, manchmal erstaunlich nah. Auch der Blick auf die Antike entzückt mich manchmal, als sähe man aus einem finstern Alpental hinaus, hinüber auf zartbesonnte ewige Inseln. Ich müsste verzweifeln, dächte ich nicht, dass wir auf finsternem gewundenem, blutigen Pfad einem neuen Humanismus dennoch uns entgegen tasten. »

${ }^{32}$ " Deutsches Lesebuch » [1922], in RA II, p. 169 : « da blickt hinter jedem einzelnen Gesicht, das uns bedeutend und aufrichtig ansieht, noch aus dunklem Spiegelgrund das rätselhafte Nationalgesicht hervor. » 
Grand Siècle français dans les décennies allant de 1750 à 1850, dont il relaie le nom de «siècle de l'esprit allemand». Dans cette attente, la constitution du canon de la littérature germanique doit s'édifier sur le modèle du canon esthétique et linguistique français :

\begin{abstract}
Nous n'avons pas un canon à la manière des Français ; comme nous ne nous retrouvons jamais dans de solides règles du jugement, le rang d'un individu, pas seulement parmi les vivants, mais même encore parmi les morts, sera toujours chez nous un rang instable. Nous avons recherché des auteurs dont la langue et le ton nous paraissaient particulièrement vrais, des auteurs chez qui l'homme entier tenait la plume. Ainsi, nous pensions saisir d'un coup ceux qui ont un cœur à eux $[\ldots]$ et en même temps, parmi eux, ceux qu'on peut appeler de vrais stylistes, de vrais prosateurs. Ils écrivaient bien parce qu'ils pensaient bien et avaient des sentiments purs, et du fait qu'ils entendent se confier eux-mêmes à nous, l'âme du peuple s'exprime en eux. ${ }^{33}$
\end{abstract}

Hofmannsthal impute à l'histoire heurtée de l'Allemagne et de l'Autriche et à l'équilibre précaire qui a régi leurs relations depuis des siècles le retard accusé par leur littérature par rapport à la littérature française, qui jouit d'une plus grande réflexivité sur ses fondements, ses procédés et sa continuité. $\mathrm{La}$ langue des auteurs classiques se voit ainsi investie de la fonction magique d'abolir les frontières du temps et l'opposition entre les individus, entre l'auteur et le peuple, entre culture classique et culture populaire. Aux yeux de Hofmannsthal, le théâtre est l'art le plus à même d'assurer cette circulation de la pensée et de la culture entre les individus et les peuples.

\title{
La tragédie racinienne, modèle esthétique et éthique
}

Grâce à sa formation classique autant qu'aux lectures qu'il approfondit tout au long de sa vie, Hofmannsthal possède une connaissance panoramique de la littérature française du XVII ${ }^{\mathrm{e}}$ siècle. Si la réception des comédies de Molière joue le rôle le plus considérable dans son théâtre, on trouve également maintes traces, dans son œuvre critique et littéraire, de sa familiarité avec les figures les plus éminentes du Grand Siècle, en particulier avec les moralistes ${ }^{34}$. Dans sa volonté de refonder le théatre germanique sur un socle classique inspiré du modèle français, Hofmannsthal ne manque du

${ }^{33}$ Ibid., p. 170 : «Wir haben nicht wie die Franzosen einen Kanon; wie wir uns nie zu festen Regeln der Beurteilung durchfinden, so wird auch der Rang des Einzelnen bei uns immer ein schwankender sein, nicht von den Lebenden, sondern sogar noch von den Toten. Wir haben solche ausgesucht, deren Sprache und Tonfall uns besonders wahr schien, solche, bei denen der ganze Mensch die Feder geführt hat. So meinten wir mit einem Griff die zu fassen, welche ein eigenes Herz haben [...], und in ihnen zugleich die, welche man wahrhafte Stilisten, wahrhafte Prosaisten nennen kann. Sie haben gut geschrieben, weil sie gut gedacht und rein gefühlt haben, und indem sie uns sich selbst auszusprechen meinen, wird das Volksgemüt in ihnen redend. »

${ }^{34}$ Voir Audrey GiBoux, Le Théâtre de Hugo von Hofmannsthal et la littérature française classique : enjeux d'une réception créatrice, thèse de doctorat en littérature comparée, université Paris-Sorbonne, 2010, p. 139-365. 
reste pas de reconnaître les tragédies françaises au rang de chefs-d'œuvre de la Weltliteratur.

Il exprime son admiration pour Corneille dès sa thèse de doctorat; la lignée qui mène le théâtre de Corneille à Hugo relie à ses yeux le peuple français à ses origines romanes, en incarnant, avec la création du Cid, un souffle hispanique sur les scènes françaises, suscitant une effervescence et un enthousiasme à la mesure de sa capacité à révéler au public français sa parenté fondatrice avec le peuple espagnol ${ }^{35}$. L'idéal chevaleresque illustré par Corneille est pour Hofmannsthal l'occasion d'une réflexion théorique sur la création théâtrale, tributaire d'une mythification de l'histoire européenne qui excède celle des littératures nationales. Le réinvestissement du passé culturel au sein d'une œuvre dramatique ne relève pas de la seule érudition, mais d'un art poétique qui conçoit la naissance des œuvres dans une sorte de libido créatrice, où les textes du passé engendrent les œuvres nouvelles, tel un organisme qui se régénère sans cesse :

Lire Corneille de cette manière [comme Hugo dans la préface de Cromwell],
s'enivrer de l'orgueil de ses opinions, de la fierté de sa diction, partager son
élément féodal, espagnol, rechercher pour ainsi dire sa fibre personnelle sous
l'enveloppe d'une forme classicisante, cela signifie s'incorporer à lui et, là où la
force géniale de l'assimilation est présente, cela signifie s'emparer de chaque
tonalité et la garder à jamais. Car c'est ainsi que les esprits d'une époque
productive s'approprient ce qui leur paraît vivant dans l'héritage des époques
antérieures. Ils sont sans érudition ; mais un désir violent de conquérir, d'enrichir
l'art en eux, les pousse dans cette direction. ${ }^{36}$

Mais si Hofmannsthal voit en Corneille le patriarche d'une lignée d'auteurs, parmi lesquels on distingue Hugo, Scribe et Sardou ${ }^{37}$, se livrant à l'exercice traditionnel de la comparaison entre les génies cornélien et racinien, il fait tourner le duel à l'avantage de Racine : il souligne certes combien Corneille sait, pour exprimer les tensions propres à la vie de cour, infiltrer au cœur même de la galanterie une dimension virile et martiale qui polarise son théâtre autour du double enjeu de l'amour et de l'honneur, mais il diagnostique dans le théâtre racinien une hybridité plus actuelle, mêlant ces deux instances opposées chez Corneille, témoignant de la complexité intime des êtres ${ }^{38}$.

35 «Studie über die Entwicklung des Dichters Victor Hugo » [1901], in RA I, p. 250.

36 Ibid., p. 298 : «In einem solchen Sinne Corneille zu lesen, an dem Hochmut seiner Gesinnung, dem Stolz seiner Diktion sich zu berauschen, sein feudales, sein spanisches Element ihm nachzufühlen, gleichsam seine persönliche Fiber unter der Hülle klassizierender Form emporzuwühlen, dies heißt sich in ihn einleben, und wo die geniale Kraft der Assimilation vorhanden ist, heißt es jenes Tones sich bemächtigen und ihn fortan zu besitzen. Denn so eignen sich die Geister einer produktiven Epoche alles an, was ihnen aus früheren Zeiten lebendig erscheint. Sie sind ohne Gelehrsamkeit; aber eine heftige Begierde, zu erobern, die Kunst in sich zu bereichern, treibt sie in jede Richtung. »

37 « Schiller» [1905], in RA I, p. 354.

${ }^{38}$ Aufzeichnungen [1925], in RA III, p. 582. 
Hofmannsthal ne cache pas, dès sa plus tendre jeunesse, combien il est sensible à la beauté de l'œuvre de Racine ${ }^{39}$, dont il loue la finesse ${ }^{40}$ et fait le symbole du combat de l'esprit français pour rejoindre la grandeur de l'Antiquitét ${ }^{41}$. Il voit en lui, aux côtés de Bossuet, le fondateur d'une autre famille d'auteurs, celle de l'esprit conservateur de l'art poétique, qui se prolonge par-delà Montesquieu et Voltaire ${ }^{42}$. La lecture assez traditionnelle qu'il donne de Racine, en opposant sa tendresse élégiaque et la pureté de son vers au souffle chevaleresque et glorieux de Corneille, semble lui avoir été soufflée par les Réflexions critiques sur quelques poètes (1746) de Vauvenargues, dont le chapitre «Corneille et Racine " proclame la supériorité de Racine sur son rival :

\begin{abstract}
Les héros de Corneille disent souvent de grandes choses sans les inspirer ; ceux de Racine les inspirent sans les dire. [...] Qu'on examine [les] ouvrages [de Racine] sans prévention : quelle facilité ! quelle abondance, quelle poésie! quelle imagination dans l'expression! Qui créa jamais une langue ou plus magnifique, ou plus simple, ou plus variée, ou plus noble ou plus harmonieuse ou plus touchante? Qui mit jamais autant de vérité dans ses dialogues, dans ses images, dans ses caractères, dans l'expression des passions ? Serait-il trop hardi de dire que c'est le plus beau génie que la France ait eu et le plus éloquent de ses poètes $?^{43}$
\end{abstract}

La noblesse retenue de l'art racinien est d'un apport étonnant dans la réflexion que mène Hofmannsthal, en temps de guerre, sur la notion de politesse, qui devient l'un des critères de sa dissociation entre les esprits allemand et autrichien, à la faveur d'un rapprochement de la politesse autrichienne avec la sociabilité française, notamment incarnée dans la déférence racinienne ${ }^{44}$. Le théâtre de Racine définit ainsi un ethos plein de réserve et de discrétion, toujours suggestif au début $\mathrm{du} \mathrm{XX}^{\mathrm{e}}$ siècle : Hofmannsthal s'en inspire pour figurer la politesse exquise et l'art de la conversation des héros de sa pièce L'Homme difficile, comme en témoignent les annotations de son édition des Nouveaux essais de critique et d'histoire de Taine, qui évoquent, sur la foi des Mémoires sur la vie de Jean Racine par son fils Louis, le goût du dramaturge pour la conversation : «Dans sa conversation il n'était jamais distrait, jamais poëte ni auteur. Il songeait moins à faire paraître son esprit que l'esprit des personnes qu'il entretenait... Il

\footnotetext{
${ }^{39}$ Hugo von HOFMANNSTHAL-Leopold von ANDRIAN, Briefwechsel, hrsg. von Walter $H$. Perl, Frankfurt am Main, Fischer, 1968, lettre de juin 1895, p. 50.

${ }^{40}$ "Ein deutscher Homer von heute », in RA I, p. 413.

${ }^{41}$ «Deutsche Festspiele zu Salzburg» [1919], in RA II, p. 256

${ }^{42}$ « Studie über die Entwicklung des Dichters Victor Hugo », in RA I, p. 247.

${ }^{43}$ Erfundene Gespräche und Briefe, p. 494. Voir Luc de VAUVENARGUES, Réflexions critiques sur quelques poètes [1746], in Réflexions, sentences et maximes morales de La Rochefoucauld, précédées d'une notice par M. Sainte-Beuve. Euvres choisies de Vauvenargues avec un choix des notes de Voltaire, Morellet, Fortia, etc. et précédées d'une notice par Suard, Paris, Garnier frères, 1867, p. 295 (édition consultée par Hofmannsthal).

${ }_{44}$ Aufzeichnungen [1915], in RA III, p. 525.
} 
vécut dans la société des femmes avec une politesse toujours respectueuse ${ }^{45} \gg$.

Si Racine est, au même titre que Corneille, un classique, Hofmannsthal souscrit cependant à cette proposition de Delacroix, qui subvertit les périodisations universitaires des mouvements esthétiques : "Racine était un romantique pour les gens de son temps. Pour tous les temps il est classique, c'est-à-dire parfait ${ }^{46}$. " Cette évocation du « romantisme racinien " s'insère dans la réflexion du peintre sur la copie en art ; extraite de son Journal (13 janvier 1857), elle est précédée de ce passage qui poursuit la comparaison de Racine et Shakespeare, récurrente depuis l'essai stendhalien du même nom de 1823. On voit qu'elle conditionne encore les interrogations hofmannsthaliennes :

Classique. À quels ouvrages est-il plus naturel d'appliquer ce nom ? C'est évidemment à ceux qui semblent destinés à servir de modèles, de règle dans toutes leurs parties. J'appellerais volontiers classiques tous les ouvrages réguliers, ceux qui satisfont l'esprit non seulement par une peinture exacte ou grandiose ou piquante des sentiments et des choses, mais encore par l'unité, l'ordonnance logique, en un mot par toutes ces qualités qui augmentent l'impression en amenant la simplicité. ${ }^{47}$

Si le classicisme de Racine est le fruit de sa perfection formelle, son œuvre représente surtout, selon Hofmannsthal, une pensée qui s'adresse au public à travers les siècles, bien qu'aient changé le goût de l'époque et l'édifice social auquel se rapportait son œuvre :

Il y a dans le rythme auquel on y traite les questions spirituelles et trouve dans le tiraillement de l'âme le chemin qui mène à une décision, dans la mesure qui établit l'équilibre entre le cœur et la tête, dans la clarté qui éclaire jusqu'à la région sombre de l'âme, un modèle éternel; les héritiers poètes peuvent s'y fier entièrement - ils sentent qu'ils demeureraient ensuite en accord avec la plus tendre conscience de soi de la nation, et ils n'ont pas besoin d'une plus haute instance. $^{48}$

${ }^{45}$ Der Schwierige. Lustspiel in drei Akten [1921], in Sämtliche Werke, BandXII, Dramen 10, hrsg. von Martin Stern in Zusammenarbeit mit Ingeborg Haase und Roland Haltmeier, Frankfurt am Main, Fischer, 1993, p. 65 et p. 545-546. Voir Hippolyte TAINE, Nouveaux essais de critique et d'histoire [1865-1901], Paris, Hachette, 1905, p. 152 (édition consultée par Hofmannsthal) et Louis RACINE, Mémoires sur la vie et les ouvrages de Jean Racine [1747], in Euvres de Jean Racine, précédées des Mémoires sur sa vie par Louis Racine, Paris, Didot, 1851, p. 16.

${ }_{46}$ Buch der Freunde, in RA III, p. 282.

47 Hofmannsthal possède l'édition suivante : Eugène DELACroIX, Literarische Werke. Deutsch von Julius Meier-Graefe, Leipzig, Insel, 1912 ; voir Journal d'Eugène Delacroix, précédé d'une étude sur le maître, par M. Paul Flat, notes et éclaircissements par MM. Paul Flat et René Piot, Paris, Plon-Nourrit, 1893-1895, vol. 3, p. 217-218.

${ }^{48}$ « Schillers Selbstcharakteristik» [1925], in RA III, p. 68 : « Es ist im Rhythmus, womit das Seelenhafte dort abgehandelt und aus dem Zwiespalt des Gemütes der Weg zu einer Entscheidung gefunden wird, im Maßgefühl, das zwischen Herz und Kopf das Gleichgewicht herstellt, in dem Klarsinn, der noch die dunkle Region der Seele aufhellt, ein ewig Vorbildliches 
Il ne s'agit pas, précise Hofmannsthal lorsqu'il souligne le caractère intemporel de la tragédie racinienne, d'en imiter servilement les formes, mais de continuer à en donner des transpositions poétiques, comme celles de Musset et Baudelaire, et de perpétuer l'idéal que représente la tendresse hérö̈que de ses personnages féminins. Si la dimension intime de l'œuvre racinienne est précisément ce qui lui octroie sa place au panthéon des grands dramaturges, Hofmannsthal prétend avoir poursuivi, dans la composition de ses livrets d'opéra, la tradition initiée par Racine et également illustrée par les drames de Claudel, dans laquelle la scène devient le lieu où se manifeste le débat intérieur des personnages ${ }^{49}$. Le conflit tragique racinien, dans sa concision, est alors rapproché des tentatives du théâtre psychologique contemporain pour sonder les motivations inconscientes de l'action :

Racine mise tout sur les décisions intérieures; qu'aurait-il besoin des décors colorés et changeants de Shakespeare ? Les quatre murs des appartements princiers, dignes mais presque nus, sont jusqu'au niveau symbolique exactement ce dont il a besoin. ${ }^{50}$

Le théâtre de Racine, révélant la psyché humaine dans une langue normée et un genre éminemment codifié, figure par son équilibre serein un modèle encore indépassé par les expérimentations du théâtre moderne et se voit ainsi rapproché, par son dénuement et sa sobriété, de la scène symboliste.

\section{Vers la fondation d'un classicisme moderne}

Hofmannsthal recompose donc une image idéale du classicisme français, érigé en modèle esthétique, moral et social. Le devoir de l'auteur moderne n'est pas, à ses yeux, de l'ordre de la commémoration respectueuse et figée des trésors du patrimoine culturel, mais de la réponse dialogique aux grands noms des lettres, fondée sur un lien généalogique et un cycle vital des œuvres en constante métamorphose ${ }^{51}$. La littérature moderne revendique son enracinement dans un passé fructueux et nullement muséifié, en vertu de sa fermentation créatrice ${ }^{52}$ : la modernité est une relecture des traditions qui lui

gegeben; diesem dürfen sich die dichterischen Nachkommen ganz anvertrauen - sie fühlen, dass sie dann mit dem zartesten Selbstbewusstsein der Nation Überein blieben, und eine höhere Instanz haben sie nicht zu kennen. »

49 « The correspondance of Hugo von HOFMANNSTHAL and Raoul AUERNHEIMER, edited by Donald G. Daviau ", Modern Austrian Literature. Journal of the International Arthur Schnitzler Research Association, n 7, 3-4 (Special Hugo von Hofmannsthal Issue), 1974, lettre du 20 décembre 1919, p. 254.

${ }^{50}$ Buch der Freunde, in RA III, p. 289 : « Racine legt alles auf innere Entscheidungen an; was sollten ihm da Shakespeares bunte und wechselnde Schauplätze; die vier Wände eines fürstlichen Gemaches, würdig aber fast kahl, sind genau bis zum Symbolischen das, was er braucht. »

51 «Zu Josef Nadlers Literaturgeschichte » [1924-1928], in RA III, p. 147.

52 « Wiener Brief IV » [1923], in RA II, p. 483-484. 
préexistent, dans un rapport herméneutique par lequel elle cherche à se légitimer.

Quoique Hofmannsthal ne se définisse jamais comme un auteur moderne, son esthétique compose avec la perte d'une harmonie dans la représentation classique du monde; sa position ne participe pas d'une essentialisation académique et atemporelle du classicisme, mais d'un classicisme moderne, qui avoue un désir d'immortalisation par l'art tout en renonçant au mirage de la perfection et de l'achèvement. La littérature classique est ainsi à la fois un héritage et un projet, où le modèle français fait figure de paradigme fécondant et de pont diplomatique entre les cultures. 